

\title{
COVALENCY EFFECTS IN HYPERFINE INTERACTIONS IN ALKALI DITHIOFERRATES
}

\author{
C. Taft, D. Raj, J. Danon
}

\section{To cite this version:}

C. Taft, D. Raj, J. Danon. COVALENCY EFFECTS IN HYPERFINE INTERACTIONS IN ALKALI DITHIOFERRATES. Journal de Physique Colloques, 1974, 35 (C6), pp.C6-241-C6-245. 10.1051/jphyscol:1974631 . jpa-00215788

\section{HAL Id: jpa-00215788 https://hal.science/jpa-00215788}

Submitted on 1 Jan 1974

HAL is a multi-disciplinary open access archive for the deposit and dissemination of scientific research documents, whether they are published or not. The documents may come from teaching and research institutions in France or abroad, or from public or private research centers.
L'archive ouverte pluridisciplinaire HAL, est destinée au dépôt et à la diffusion de documents scientifiques de niveau recherche, publiés ou non, émanant des établissements d'enseignement et de recherche français ou étrangers, des laboratoires publics ou privés. 


\title{
COVALENCY EFFECTS IN HYPERFINE INTERACTIONS IN ALKALI DITHIOFERRATES
}

\author{
C. A. TAFT $\left({ }^{1}\right)$, D. RAJ and J. DANON \\ Centro Brasileiro de Pesquisas Físicas, Rio de Janeiro, Brazil
}

\begin{abstract}
Résumé. - Les spectres Mössbauer des composés tétraédriques (sp $\left.{ }^{3}\right)$ de $\mathrm{Fe}(\mathrm{III})$ de formule générale $\mathrm{AFeS}_{2}(\mathrm{~A}=\mathrm{K}, \mathrm{Rb}, \mathrm{Cs})$ ont été relevés entre $4,2 \mathrm{~K}$ et $300 \mathrm{~K}$. RbFeS $\mathrm{R}_{2}$ et $\mathrm{CsFeS}_{2}$ présentent des structures hyperfines magnétiques à des températures plus basses que $\mathrm{KFeS}_{2}$, ce que nous avons attribué à des effets de liaison. L'hybridation tétraédrique dans ces composés facilite l'utilisation des calculs des gradients de champ électrique, des différences d'électronégativité et des constantes d'étalonnage du déplacement isomérique pour déterminer la fraction de la densité des électrons $4 \mathrm{~s}$ au site du noyau ${ }^{57} \mathrm{Fe}$. Des modèles simples de covalence permettent d'estimer l'ordre de grandeur des champs magnétiques internes et de relier les variations des paramètres hyperfins au caractère covalent des liaisons.
\end{abstract}

\begin{abstract}
Mössbauer spectra were taken of a series of compounds with tetrahedrally coordinated ( $\mathrm{sp}^{3}$ ) iron (III) having a general formula $\mathrm{AFeS}_{2}$, where $\mathrm{A}=\mathrm{K}, \mathrm{Rb}, \mathrm{Cs}$ over a temperature range 4.2-300 K. RbFeS ${ }_{2}$ and $\mathrm{CsFeS}_{2}$ exhibited magnetic hyperfine structures at lower temperatures than that observed for $\mathrm{KFeS}_{2}$, which we have correlated with bonding effects. The tetrahedral hybridization in these compounds facilitates the use of EFG calculations, electronegativity differences and isomer shift calibration constants, for determining the fractional $4 \mathrm{~s}$ electron densities at the ${ }^{57} \mathrm{Fe}$ nucleus. Simple covalency models are used to make order of magnitude estimates of the internal magnetic fields and for correlating the spread of hyperfine parameters with the covalent character of the bonds.
\end{abstract}

1. Introduction. - It has been often emphasized that the effects of covalency in the hyperfine interactions may be quite important [1-7]. Many theoretical calculations have also been made in order to determine the parameters which are relevant for interpreting the experimental data. It is interesting, however, that recent unrestricted Hartree Fock calculations [7] of isomer shift calibration constant for ${ }^{57} \mathrm{Fe}$ are in agreement with the values determined by one of us using a much simpler model [1,2].

We have thus emphasized in this paper the use of simple available models for determining the amount of covalency in tetrahedrally bonded iron compounds $\left(\mathrm{KFeS}_{2}, \mathrm{RbFeS}_{2}\right.$ and $\left.\mathrm{CsFeS}_{2}\right)$ and the effects of this bonding on the measured hyperfine parameters.

The bonding properties in these compounds are reasonably well describe $\mathrm{d}$ by assuming $4 \mathrm{sp}^{3}$ hybridization, the participation of $3 \mathrm{~d}$ electrons in the bonding being of less importance. This has been emphasized to be true at least in very covalent crystals and for tetrahedral coordination, where sizable reduction of the hyperfine fields are accompanied by small $3 \mathrm{~d}$ covalency [5].

This type of bonding scheme $\left(3 \mathrm{~d}^{5}-4 s \mathrm{p}^{3}\right)$ which has been propose $d$ for these compounds, in which high spin

(1) On leave from the Physics Department, University of Panama, Panama. ferric iron appears to maintain it's S-state symmetry [5, 8-11] facilitates the interpretation of our experimental results in terms of simple covalency models such as the Walker-Wertheim-Jaccarino proposed model [4].

2. Structure and experimental. $-\mathrm{KFeS}_{2}$ grows in permanganate colored needle-shaped monoclinic crystals with space-group symmetry $\mathrm{C}_{2 \mathrm{~h}}^{6}$ with four molecules per unit cell having all $\mathrm{Fe}$ atoms at equivalent sites. The structure $[8,9]$ of $\mathrm{RbFeS}_{2}$ is analogous to that of $\mathrm{KFeS}_{2}$ whereas $\mathrm{CsFeS}_{2}$ forms orthorhombic crystals with space group $D_{2 \mathrm{~h}}^{25}$ with a tetramolecular unit cell. In all these compounds the iron atoms are principally covalently bonded by nearly regular sulphur tetrahedra. The $\left(\mathrm{FeS}_{2}\right)^{-}$ions are arranged in chains of $\mathrm{FeS}_{4}$ tetrahedra linked by their edges with short $\mathrm{Fe}-\mathrm{Fe}$ distances.

The specimens were prepared $[8,9]$ from A. R. quality chemicals by fusing iron powder with the corresponding alkali carbonate and sulphur and leaching the cold product in water. Our samples were characterized by X-ray examination.

Mössbauer spectra of polycrystalline sample were recorded in the standard transmission geometry with a constant acceleration Doppler velocity transducer, 400 multichannel analyzer and $\mathrm{Co}^{57}$ in $\mathrm{Pd}$ matrix with an activity of $25 \mathrm{mCi}$. The low temperature measure- 
ments for $\mathrm{RbFeS}_{2}$ were recorded using an Elscint cryostat with temperature controller. The temperature stability was $\pm 2^{\circ} \mathrm{C}$. The cryogenic arrangement for the liquid helium measurement employed a Sulfrian cryostat. The lines were fitted by least square analysis on an IBM 370 computer.

3. Calculations of effective charges and $4 s$ electron densities. - The values of isomer shift and temperature independent quadrupole splitting in $\mathrm{KFeS}_{2}$, $\mathrm{RbFeS}_{2}$ and $\mathrm{CsFeS}_{2}$ are typical of high spin ferric iron in which the $3 \mathrm{~d}^{5} \mathrm{~S}$-state symmetry of $\mathrm{Fe}^{3+}$ does not

\section{TABLE I}

Experimental measurements of hyperfine parameters

$\begin{array}{lccc}\text { Compound } & \begin{array}{c}\text { Isomer shift } \\ (\mathrm{mm} / \mathrm{s})\left({ }^{a}\right)\end{array} & \begin{array}{c}\text { Quadrupole } \\ \text { splitting } \\ (\mathrm{mm} / \mathrm{s})\left({ }^{b}\right)\end{array} & \begin{array}{c}\text { Magnetic field } \\ (\mathrm{kG})\left({ }^{(}\right)\end{array} \\ - & - & - & - \\ \mathrm{KFeS}_{2} & 0.37 & 0.53 & 215 \\ \mathrm{RbFeS}_{2} & 0.34 & 0.45 & 196 \\ \mathrm{CsFeS}_{2} & 0.31 & 0.43 & 150\end{array}$

(a) Saturation values (w. r. t. Fe). Experimental errors are $\pm 0.02 \mathrm{~mm} / \mathrm{s}$.

${ }^{(b)}$ Temperature independent values. Experimental errors are $\pm 0.02 \mathrm{~mm} / \mathrm{s}$.

(c) Saturation values. Experimental errors are $\pm 5 \mathrm{kG}$.

appear to be significantly affected by covalent bonding [5, 8-11]. It thus appears reasonable to describe these compounds by a simple model of effective charges at all lattice sites which are required to produce the measured field gradient at the Fe nucleus, subject to the additional constraints imposed by the charge neutrality condition and the monovalent character of the cations.

Using the available crystallographic data $[8,9]$ the nine components of the EFG tensor are calculated from the relation

$$
V_{i j}^{\prime}=\sum_{k} e_{k}\left(3 R_{i k} R_{j k}-\delta_{i j} R_{k}^{2}\right) / R_{k}^{5} \quad(i, j=x, y, z)
$$

where $e_{k}$ and $\left(R_{x k}, R_{y k}, R_{z k}\right)$ are the effective charges and the coordinates of the $k$ th atom in an arbitrary orthogonal coordinate system [12]. The EFG tensor thus obtained was diagonalized and the principal values of $V_{i j}$ were designated according to the convention that

$$
\begin{gathered}
\left|V_{z z}\right|>\left|V_{y y}\right|>\mid V_{x x} \\
e q=V_{z z} \text { and } \eta=\left(V_{x x}-V_{y y}\right) / V_{z z} .
\end{gathered}
$$

These values and the latest values $[13,14]$ of $Q$ and $\gamma_{\infty}$ are used to calculate $\Delta E_{Q}$ from the relation

$$
\Delta E_{Q}=\frac{1}{2} e^{2} q Q\left(1+\eta^{2} / 3\right)^{1 / 2}\left(1-\dot{\gamma}_{\infty}\right) .
$$

The lattice sum calculations were carried out on an IBM 370 computer within a chosen radial distance from the central iron and this radius of summation was systematically varied until the convergence was attained. Variations were also not significant when the uncertainties of the structure were taken into account. All our final results were calculated within a sphere of $50 \AA$ radius.

The electronegativity difference values, and comparison of the sum of ionic radius of $K, R b, C$ s and $S$ with the observed bond length, indicates that the alkali metals in these compounds are ionic in character $[8,9$, 15]. We have therefore assigned charge +1.0 to $\mathrm{K}, \mathrm{Rb}$ and $\mathrm{Cs}$, a wide range of effective charges to iron and the corresponding charges on sulphur were determined by the charge neutrality condition.

Our calculations yield a best fit with our experimentally determined values of quadrupole splitting when we assign charges of approximately $1.61,1.46$ and 1.31 (units of electron charge) to iron in $\mathrm{KFeS}_{2}$, $\mathrm{RbFeS}_{2}$ and $\mathrm{CsFeS}_{2}$ respectively.

Assuming each of the $4 \mathrm{sp}^{3}$ tetrahedral bonds as equivalent the fractional 4 s electron densities at the $\mathrm{Fe}$ nucleus in each compound can be determined from

$$
4 s=(3.0-\text { E. F. }) / 4
$$

where E. F. are the effective charges on iron previously determined. These estimates yield electron densities on the order of $0.35-0.42$ in these three compounds.

The total s-electron density at the ${ }^{57} \mathrm{Fe}$ nucleus in these compounds may be written as the sum of the inner-shell contribution and a fraction $x \psi_{4 s}^{2}(0)$ of $4 \mathrm{~s}$ density arising from the partial occupation of this shell by electrons from the ligands [4]

$$
\psi_{s}^{2}(0)=\sum_{1}^{3} \psi_{\text {is }}^{2}(0)+x \psi_{4 s}^{2}(0) .
$$

The I. S. is proportional to the total s electron density $\psi_{\mathrm{s}}^{2}(0)$. The proportionality constant

$$
\alpha=-0.2 a_{0}^{-3} \mathrm{~mm} / \mathrm{s}
$$

was determined $[1,2]$ from models based on electronegativity differences and molecular orbital calculations and is in agreement with more recent evaluations [7].

The $4 s$ densities thus calculated $[1,2]$ for the alkalidithioferrates, using the experimental values of isomer shift are on the order of $0.35-0.40$. These values are in the range given by our above estimates.

Ferreira [16] has also suggested a model for determining the charge transfer in complex molecules which is based on molecular orbital calculations, and in addition to electronegativity differences and coordination numbers also takes into account the principle of equalization of effective electronegativities and electronic screening constants.

Following the notation in Ferreira's paper [16], the 
charge transfer from the sulphur ligands in these compounds can be determined from equations

$$
\begin{aligned}
& X_{\mathrm{B}}(E)=X_{\mathrm{B}}(0)+q \sum_{i}^{m} \Delta X_{i}^{+}, \\
& X_{\mathrm{A}}(E)=X_{\mathrm{A}}(0)-q \sum_{i}^{n} \Delta X_{j}^{-}
\end{aligned}
$$

and

$$
q=\frac{X_{\mathrm{B}}(0)-X_{\mathrm{A}}(0)}{\sum_{1}^{m} \Delta X_{i}^{+}+\sum_{1}^{n} \Delta X_{j}^{-}}
$$

in which $\left(X_{\mathrm{A}}(E), X_{\mathrm{B}}(E)\right)$ and $\left(X_{\mathrm{B}}(0), X_{\mathrm{A}}(0)\right)$ are electronegativities of atoms $A$ and $B$ in the combined and uncombined states respectively, $n$ and $m$ are the corresponding oxidation numbers, $q$ is the fractional electron charge transferred, and $\Delta X_{i}^{+}$and $\Delta X_{j}^{-}$represent the variation in electronegativities as fractional electrons are transferred from one atom to another. The expressions for $\Delta X_{i}^{+}$and $\Delta X_{j}^{-}$are given by

$$
\begin{aligned}
& \Delta X_{i}=\frac{\sigma_{i}}{1-\sigma_{k}}\left(X_{\mathrm{B}+1}(0)-X_{\mathrm{B}}(0)\right) \\
& \Delta X_{j}^{-}=\frac{\sigma_{j}}{1-\sigma_{k}}\left(X_{\mathrm{A}}(0)-X_{\mathrm{A}-1}(0)\right)
\end{aligned}
$$

where $\sigma_{i}, \sigma_{j}, \sigma_{k}$ are screening constants [16].

The charge transfer for the alkali dithioferrates determined [15-17] from these equations yield $4 \mathrm{~s}$ electron densities on the order of $0.36-0.39$. These estimates support our previous results.

One must not be carried away, however, by the agreement obtained from these independent estimates since we have used the simplest of theoretical models available and must also make allowances for the uncertainties in our experimental measurements and coupling constants used in these calculations. The most we can conclude from such estimates is that they suggest a small spread of rather significant iron covalency in the alkali dithioferrates. These estimates will also be used to correlate the unusually small values of internal magnetic fields with the covalent character of the bonds.

It has been noted [1-7] that the hyperfine parameters are usually larger for more ionic compounds as compared to those which are more covalently bonded. This is in conformity with our experimental measurements (Table I) which indicate that the larger values of hyperfine parameters correspond to the more ionic compounds. It would thus appear that the larger cations in $\mathrm{RbFeS}_{2}$ and $\mathrm{CsFeS}_{2}$ tend to force the $\mathrm{Fe}$ and $\mathrm{S}$ atoms into closer bonds. Such a comparison is at least reasonable for isostructural compounds and would explain the smaller values of hyperfine parameters and $\mathrm{Fe}$-S distance in $\mathrm{RbFeS}_{2}$ as compared to the analogously structured compound $\mathrm{KFeS}_{2}$.
4. Analysis of the low temperature Mössbauer spectroscopic data. - In figures 1 and 2 we have plotted the low temperature Mössbauer spectroscopic data for $\mathrm{RbFeS}_{2}$ and $\mathrm{CsFeS}_{2}$. The Mössbauer spectra for $\mathrm{RbFeS}_{2}$ from room temperature down to liquid nitrogen confirms magnetic ordering at about $-88^{\circ} \mathrm{C} \pm 2^{\circ} \mathrm{C}$ and shows a significant increase in isomer shift on passing the transition point.



FIg. 1. - Mössbauer spectrum of $\mathrm{CsFeS}_{2}: a$ ) at room temperature, $b$ ) at liquid helium temperature.

If the isomer shift $(\delta)$ is assumed to be independent of temperature $-\partial \delta / \partial T$ is proportional to the specific heat. The deviation below the transition point may be explained by a temperature dependent component of the isomer shift which is approximately proportional to the magnetic field.

The Mössbauer spectra also indicates relaxation effects near the transition point. Similar effects have been observed for iron (III) antiferromagnetically ordered compounds [18].

The liquid helium measurement for $\mathrm{CsFeS}_{2}$ also indicated the appearance of a magnetic hyperfine interaction. The transition point is below liquid nitrogen temperature.

Magnetic susceptibility measurements and crystal structure analysis suggest that $\mathrm{Fe}$ is antiferromagnetically coupled in these compounds [9]. For interactions between cations in tetrahedral sites correlation superexchange and cation-cation interactions are both unambiguously antiferromagnetic for $\mathrm{d}^{5}-\mathrm{d}^{5}$ interactions [6]. In $\mathrm{CuFeS}_{2}$, which is antiferromagnetic $\mathrm{Fe}$ and $S$ have the same type of bonding scheme and similar crystal structure as the alkali-dithioferrates [8].

For $\mathrm{Fe}-\mathrm{Fe}\left(\mathrm{d}^{5}\right)$ interactions, the Anderson's exchange integral is given by $J_{i j}=-2 b_{i j} / 4 S^{2} U$, where 


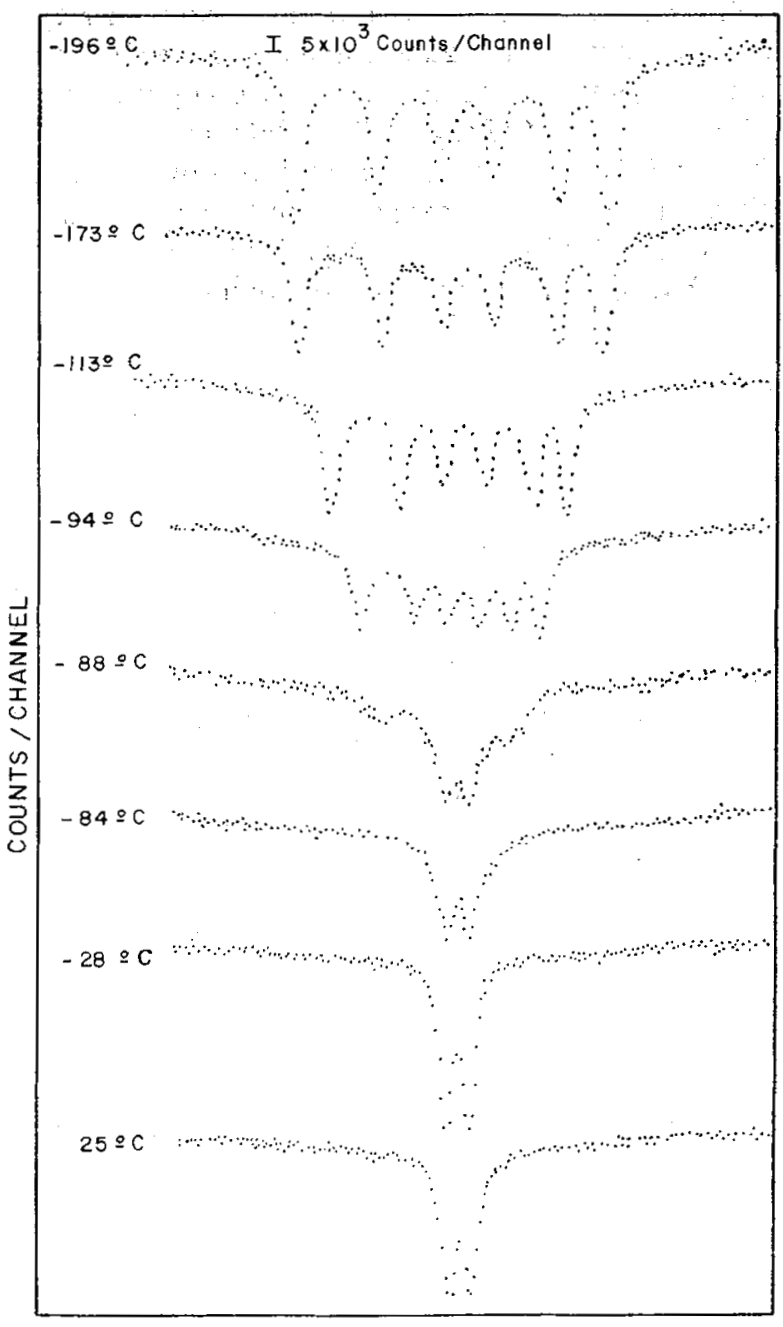

FIG. 2. - Mössbauer spectrum of $\mathrm{RbFeS}_{2}$ from room temperature down to liquid nitrogen temperature.

$S=\frac{5}{2}$ and $U$ is the electrostatic energy. The dominant parameter $b_{i j}$ is proportional to the orbital overlap and increases exponentially with decreasing $\mathrm{Fe}-\mathrm{Fe}$ separation [6]. This would explain the lower transition temperatures in $\mathrm{RbFeS}_{2}$ and $\mathrm{CsFeS}_{2}$ in which the $\mathrm{Fe}-\mathrm{Fe}$ distances are correspondingly larger than in $\mathrm{KFeS}_{2}$ which has a higher transition temperature $\left(-28^{\circ} \mathrm{C}\right)[10]$.

Our previous EFG calculations in the alkali-dithioferrates indicated small values of asymmetry parameter on the order of 0.09 . Consequently, if we neglect direct magnetic and magnetostrictive effects on the quadrupole coupling, the angle between the internal magnetic field and the symmetry axis of the EFG tensor can be determined from the relation [2]

$$
\left(3 \cos ^{2} \theta-1\right)=8 \varepsilon / e^{2} q Q
$$

where $e^{2} q Q$ can be obtained from our quadrupole splitting values and the parameter $\varepsilon$ can be deduced from the line positions in the Mössbauer spectrum. The estimated values are in the range $18^{\circ}-20^{\circ}-24^{\circ}$.
The measured saturation values for the alkalidithioferrates are: $\mathrm{KFeS}_{2}(215 \pm 5) \mathrm{kG}$, $\mathrm{RbFeS}_{2}$ $(196 \pm 5) \mathrm{kG}, \mathrm{CsFeS}_{2}(150 \pm 5) \mathrm{kG}$. The saturation values for the hyperfine fields in these compounds are unusually small for a ferric ion. These compare however with the values found in some covalent materials and are much smaller than the ionic value of $630 \mathrm{kOe}[3]$.

Apparently there are considerably covalency effects perhaps also negative contributions from neighboring spins. Zero point motion of the spins cannot account for such a large reduction [18]. It has also been shown that the apparent percentage of delocalization of the antibonding $3 \mathrm{~d}$ electrons in the ${ }^{6} \mathrm{~S}_{5 / 2}$ state to the neighboring atoms is generally very small and cannot be the primary cause for the reduction of the hyperfine fields [5].

The observed values of hyperfine fields are appreciably larger for ionic crystals than for covalently bonded ones and it has been often emphasized that the magnitude of the internal field in an iron (III) complex is a good indication of the extent of covalency, the more covalent the bonds, the smaller the observed hyperfine fields $[1-7,19-20]$. This would certainly explain the correspondingly smaller fields in $\mathrm{RbFeS}_{2}$ and $\mathrm{CsFeS}_{2}$ which are more covalently bonded.

It was thus considered of interest to make order of magnitude estimates of these fields in the alkalidithioferrates using the available covalency models.

The spread of the hyperfine fields have been correlated with the covalent-ionic character of the bonds by using models based on electronegativity differences [19, 20]. These correlations suggest that one can make order of magnitude estimates of the hyperfine field in a given compound by considering the varying covalent character of the metal-ligand neighbor bonds via electronegativity differences.

Like the alkali-dithioferrates, $\alpha-\mathrm{NaFeO}_{2}$ [21] and $\mathrm{CuFeO}_{2}$ [22] are ternary compounds having iron in high spin trivalent $3 d^{5}{ }^{6} \mathrm{~S}_{5 / 2}$ state and the low temperature magnetic fields (saturation values) observed are $455 \pm 5 \mathrm{kOe}$ and $520 \pm 5 \mathrm{kOe}$. Using these saturation values and the models of Pauling and Hannay and Smyth [17], with due consideration for electronegativity differences and coordination numbers, the covalent character of the $\mathrm{FeS}_{2}^{-}$and $\mathrm{FeO}_{2}^{-}$clusters are used to estimate the hyperfine fields which lie in the range from 200 to $244 \mathrm{kOe}$. These values are a little high for some of our measurements but are certainly of the right order of magnitude.

Molecular orbital calculations suggest that there should be a linear relationship between the covalent induced hyperfine field and the charge transfer to the $4 \mathrm{~s}$ state [5].

Assuming such a relationship, we can write

$$
H_{\mathrm{hf}}=H^{\text {ionic }}-\Delta H, \quad \Delta H=Q_{4 \mathrm{~s}} \times H_{4 \mathrm{~s}}
$$

where $H^{\text {ionic }}=630 \mathrm{kOe}$ is the ionic field and $H_{4 \mathrm{~s}}=1348 \mathrm{kG}$ is the average hyperfine field due to 
unpaired $4 \mathrm{~s}$ electrons [3]. $Q_{4 \mathrm{~s}}=0.35-0.40$ is the $4 \mathrm{~s}$ contribution from the sulphur ligands previously determined.

The estimated hyperfine field lies in the range from 100 to $160 \mathrm{kG}$, which is a reasonable result considering the simplicity of our model. A more proper estimate should take into consideration other contributions, such as the $4 \mathrm{~s}$ radial polarization which is at present less accessible.

We have previously determined from EFG calculations the effective charges on iron in the alkalidithioferrates. Other estimates of effective charges can be obtained from the $4 s$ electron densities i. e. E. F. $=3.0-4 \times Q_{4 \mathrm{~s}}$. Using these values of effective charges and assuming that the fields are proportional $[3,5,19-20,23]$ to ionic character (E. F. $/ 3.0)$ of $\mathrm{Fe}^{3+}$, the estimated spread of the internal fields lie in the range $160-216 \mathrm{kG}$ and is in good agreement with the measured values. Our experimental measurements lie in the total range obtained from these three independent estimates (Table II).

We would like to emphasize the fact that these results were obtained by using very simple models and have not by far exhausted all possible contributions. However, we would like to note, that in the absence of more exact and sophisticated calculations, the uncertainties involved in these estimates are well compensated by the realistic insight into the physical differences of the environments provided by such simple models.
TABLE II

Order of magnitude estimates of internal magnetic fields in $\mathrm{KFeS}_{2}, \mathrm{RbFeS}_{2}$ and $\mathrm{CsFeS}_{2}$



$\left({ }^{a}\right)$ Using the models of Pauling and Hannay and Smyth (ref. [17]).

$\left(^{b}\right)$ Using model based on molecular orbital calculations (ref. [5]).

(c) Using I. S. proportionality constant, EFG calculations and Ferreira's model (ref. [1-4], [16-20]).

The limited but rather satisfying results discussed in this paper suggest the possibility of characterizing an isostructural series of compounds by one well defined covalency parameter, which would serve to link the various hyperfine interactions, facilitating the interpretation of experimental measurements.

Acknowledgments. - We would like to acknowledge stimulating discussions with Dr. A. A. Gomes. Financial aid from OEA and CNPq (Brasil) is also gratefully acknowledged.

\section{References}

[1] Danon, J., Tech. Rept. Ser. Intern. At. Energy Agency 50 (1966) 89.

[2] Danon, J., In Chemical applications of Mössbauer Spectroscopy (Edited by Goldanskii, V. I. and Herber, R. H.) (Academic Press, New York) 1968.

[3] Watson, R. E. and Freeman, A. J., Phys. Rev. 123 (1961) 2027.

[4] Walker, L. R., Wertheim, G. K. and Jaccarino, V., Phys. Rev. Lett. 6 (1961) 98.

[5] Simanek, E. and Muller, K. A., J. Phys. Chem. Solids 31 (1970) 1027.

[6] Goodenough, J. B., Magnetism and the Chemical Bond (Edited by Cotton, F. A.) (Interscience Publishers, New York) 1963.

[7] Duff, K. J., Phys. Rev. B 9 (1974) 66.

[8] Boon, J. W. and MacGillavry, C. H., Rec. Trav. Chim. 61 (1942) 910

[9] BRonger, W., Z. Anorg. Allg. Chem. 359 (1968) 225.

[10] Kerler, W., Neuwirth, W., Fluck, E., Kugh, P. and ZiMMERMAN, B., $Z$. Phys. 173 (1963) 321.

[11] Raj, D. and Puri, S. P., J. Chem. Phys. 50 (1969) 3184.
[12] TAFT, C. A., RAJ, D. and Danon, J. (to be published in Phys. Stat. Sol. B)

[13] Mössbauer Effect Data Index, Edited by Stevens, J. G. and Stevens, V. E. (Plenum Data Corporation, New York) 1971

[14] Sternheimer, R. M., Phys. Rev. 130 (1963) 1423.

[15] Pauling, L., The Nature of the Chemical Bond (Cornell Univ. Press, 3rd ed., New York) 1960.

[16] Ferreira, R., Trans. Faraday Soc. 59 (1963) 1064.

[17] Pritchard, H. O. and Skinner, H. A., Chem. Rev. 55 (1955) 745 .

[18] Stampfel, J. P., Oosterhuis, W. T., Window, B. and Barros, F. S., Phys. Rev. B 8 (1973) 4371.

[19] Title, R. S., Phys. Rev. 132 (1963) 623.

[20] Matamura, O., J. Phys. Soc. Japan 14 (1959) 108.

[21] Ichida, T., Shinjo, T., Bando, Y. and TaKada, T., $J$. Phys. Soc. Japan 29 (1970) 79.

[22] Muir, A. H. and Wiedersich, H., J. Phys. Chem. Solids 28 (1967) 65.

[23] Van Wierengen, J. S., Discussions Faraday Soc. 19 (1955) 118. 